\title{
Shortcut to Geostrophy in Wave-Driven Rotating Turbulence: The Quartetic Instability
}

\author{
Maxime Brunet, ${ }^{1}$ Basile Gallet, ${ }^{2}$ and Pierre-Philippe Cortet $\odot^{1, *}$ \\ ${ }^{1}$ Université Paris-Saclay, CNRS, FAST, 91405 Orsay, France \\ ${ }^{2}$ Université Paris-Saclay, CNRS, CEA, Service de Physique de l'État Condensé, 91191 Gif-sur-Yvette, France
}

(Received 29 July 2019; accepted 2 March 2020; published 24 March 2020)

\begin{abstract}
We report on laboratory experiments of wave-driven rotating turbulence. A set of wave makers produces inertial-wave beams that interact nonlinearly in the central region of a water tank mounted on a rotating platform. The forcing thus injects energy into inertial waves only. For moderate forcing amplitude, part of the energy of the forced inertial waves is transferred to subharmonic waves, through a standard triadic resonance instability. This first step is broadly in line with the theory of weak turbulence. Surprisingly however, stronger forcing does not lead to an inertial-wave turbulence regime. Instead, most of the kinetic energy condenses into a vertically invariant geostrophic flow, even though the latter is unforced. We show that resonant quartets of inertial waves can trigger an instability — the "quartetic instability" — that leads to such spontaneous emergence of geostrophy. In the present experiment, this instability sets in as a secondary instability of the classical triadic instability.
\end{abstract}

DOI: 10.1103/PhysRevLett.124.124501

Introduction.-Turbulence subject to global rotation [1-3] can take multiple forms depending on the large-scale mechanism driving it. At the extremes of the spectrum are the Taylor-Proudman theorem [4], which states that rapidly rotating flows tend to become two-dimensional (2D, or "geostrophic," invariant along the rotation axis) and weak turbulence theory (WTT) [5], which describes the flow as a collection of nonlinearly interacting inertial waves. Both approaches address the low-Rossby-number limit, but they are somewhat blind to one another: the Taylor-Proudman theorem is established for a slowly evolving flow, thus ruling out inertial waves at the outset. On the other hand, inertial-wave turbulence assumes that there is no geostrophic flow to begin with. The flow is then protected from the emergence of geostrophy, because triadic interactions of inertial waves cannot transfer energy from the fast wave modes to the slow geostrophic one $[1,6]$ : at this order of the low-Rossby-number asymptotic expansion, the geostrophic component remains zero.

A prerequisite to testing WTT in the laboratory is to be able to generate an ensemble of nonlinear inertial waves. Because this is an arduous task, most rotating turbulence experiments use instead standard forcing devices of nonrotating turbulence: grids [7-10], jets [11-14], vortex generators [15,16], and impellers [17]. These devices are rather inefficient at forcing inertial waves, either because they lack the right time dependence to match the wave dispersion relation (most of them are steady), or because they are directly compatible with a Taylor-Proudman-like 2D flow. The geostrophic component then dominates the flow in the low-Rossby-number limit, with a small energy fraction in the wave modes $[13,14,16,18]$.
To investigate nonlinear wave dynamics, there is thus a crucial need for experiments with energy input into the wave modes only. Experimental methods have arisen to generate isolated inertial-wave beams in rotating flows [19-24], but the fate of a chaotic ensemble of inertial waves remains unexplored in the laboratory. In this Letter, we report on a rotating turbulence experiment where energy is input into the wave modes only, with the following questions in mind: what happens beyond the first triadic subharmonic instability? Is inertial-wave turbulence really protected from the emergence of a strong geostrophic mode? If not, what is the physical mechanism responsible for the emergence of geostrophy?

Experimental setup.-The experimental setup is sketched in Fig. 1. Thirty-two horizontal cylinders of diameter $d=4 \mathrm{~cm}$ oscillate vertically inside a parallelepipedic water

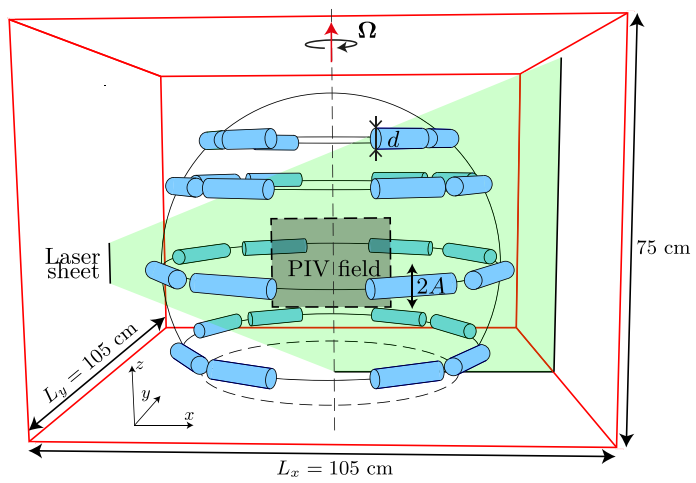

FIG. 1. Experimental setup. 32 horizontal cylinders oscillate vertically inside a tank centered on a platform rotating at $\Omega=18 \mathrm{rpm}$. 
tank of $105 \times 105 \mathrm{~cm}^{2}$ base and $75 \mathrm{~cm}$ height, with an amplitude $A$, an angular frequency $\omega_{0}$, and independent random initial phases. The cylinders are tangent to an 80 -cm-diameter virtual sphere horizontally centered in the water tank.

The apparatus is mounted on a two-meter-diameter platform rotating at a rate $\Omega=18 \mathrm{rpm}$ around the vertical axis $z$. The cylinders oscillate at $\omega_{0}=0.84 \times 2 \Omega$, each cylinder generating four inertial-wave beams $[23,25]$ making angles $\pm \arccos \left(\omega_{0} / 2 \Omega\right) \simeq \pm 32.9^{\circ}$ with the horizontal [4]. In the central region of the sphere, these beams interact nonlinearly, producing a homogeneous turbulent flow. The amplitude of oscillation $A$ is varied from 1.0 to $8.2 \mathrm{~mm}$, which corresponds to a forcing Reynolds number $130 \leq$ $\operatorname{Re}=A \omega_{0} d / \nu \leq 1040$ and a forcing Rossby number $0.022 \leq \mathrm{Ro}=A \omega_{0} / 2 \Omega d \leq 0.172$. We access the velocity field inside a vertical plane containing the center of the sphere with an on-board particle image velocimetry (PIV) system. Two velocity components $\left(u_{x}, u_{z}\right)$ are measured with a resolution of $1.94 \mathrm{~mm}$ over an area $\Delta x \times \Delta z=$ $285 \times 215 \mathrm{~mm}^{2}, 12$ times per wave maker period $T=2 \pi / \omega_{0}$.

Triadic resonance instability.-We show in Fig. 2 the temporal energy spectrum of the velocity field in the statistically steady state. For the lowest amplitude $(\operatorname{Re}=130)$, the spectrum displays two dominant peaks, the next subdominant peaks being less energetic by 2 orders of magnitude. A first peak at normalized frequency $\omega_{0}^{*}=$ $\omega_{0} / 2 \Omega=0.84$ corresponds to the waves forced by the wave makers, while a second one at $\omega^{*}=\omega / 2 \Omega=0.5$ corresponds to a "precession flow" induced by Earth's rotation $[26,27]$. This spectrum illustrates the base flow at low forcing amplitude: an ensemble of wide inertial wave

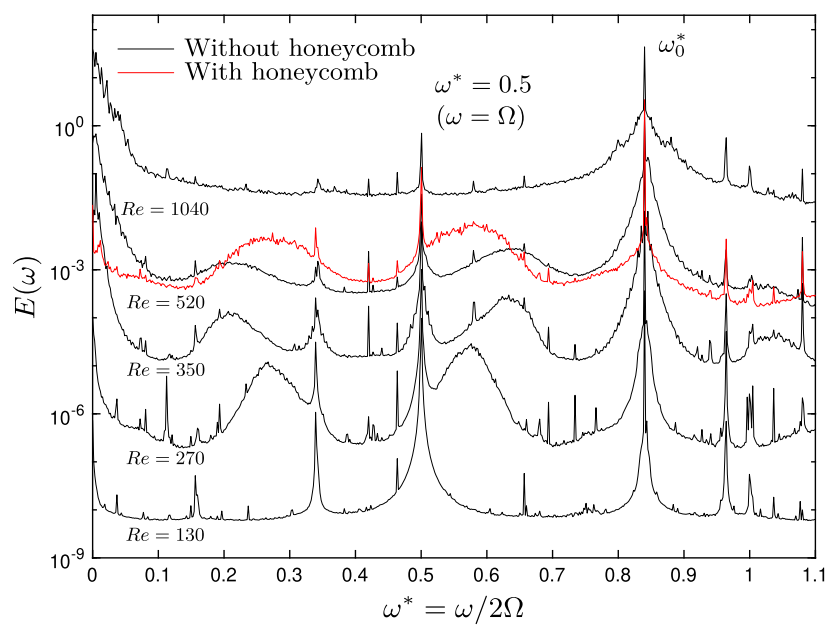

FIG. 2. Temporal spectrum of the velocity field. A vertical shift by a factor of 10 has been introduced between successive spectra. The red spectrum corresponds to an additional experiment at $\operatorname{Re}=520$ with a honeycomb bottom boundary. beams generated by the wave makers and propagating towards the central region (see the velocity fields in Fig. 3).

Increasing the forcing amplitude to $\mathrm{Re}=270$, two subharmonic bumps emerge around frequencies $\omega_{1}^{*}=$ $0.26 \pm 0.04$ and $\omega_{2}^{*}=0.58 \pm 0.04$. They correspond to inertial waves created by the triadic resonance instability of the primary waves, following the classical scenario reported in various experimental [21,24,28,29] and numerical [30-32] studies. The secondary frequencies notably satisfy a resonance condition with the primary waves: $\omega_{1}^{*}+\omega_{2}^{*} \simeq \omega_{0}^{*}=0.84$ [33].

Emergence of geostrophy.-In parallel, we observe the emergence of a strong peak at $\omega^{*}=0$ in the high-Re spectra. Low-pass filtering the velocity field with $\omega^{*}<$ 0.10 reveals that this peak is associated with $2 \mathrm{D}$ geostrophic vortices wandering in the horizontal plane. The snapshots in Fig. 3 highlight this emergence of geostrophy.

In Fig. 3, we show time series of the kinetic energies $K_{2 \mathrm{D}}(t)$ in the vertically averaged velocity field inside the PIV domain, $K_{\text {lf }}(t)$ in the low-frequency mode (using a low-pass filter with $\left.\omega^{*}<0.1\right), K_{\mathrm{sh}}(t)$ in the first subharmonic bump produced by the triadic instability (band-pass filter with $\left.0.1<\omega^{*}<\omega_{0}^{*} / 2\right)$ and $K_{\omega_{0}}(t)$ in the forced waves (band-pass filter with $\left|\omega^{*}-\omega_{0}^{*}\right|<0.1$ ). The time series of $K_{2 \mathrm{D}}$ and $K_{\mathrm{lf}}$ are almost identical, the difference being due to some pollution of $K_{2 \mathrm{D}}$ by the large-scale precession-induced flow at $\omega^{*}=0.5$. This confirms that the low-frequency spectrum corresponds to the $2 \mathrm{D}$ geostrophic flow. Most interestingly, Fig. 3 highlights the bifurcation sequence by which the geostrophic flow arises: (i) For $\operatorname{Re}=130$, the flow consists mostly in primary waves, with very low levels of $K_{\mathrm{lf}}$ and $K_{\mathrm{sh}}$. (ii) For $\mathrm{Re}=270$, the system is above the threshold of the triadic instability, with significant subharmonic energy $K_{\mathrm{sh}}$ and
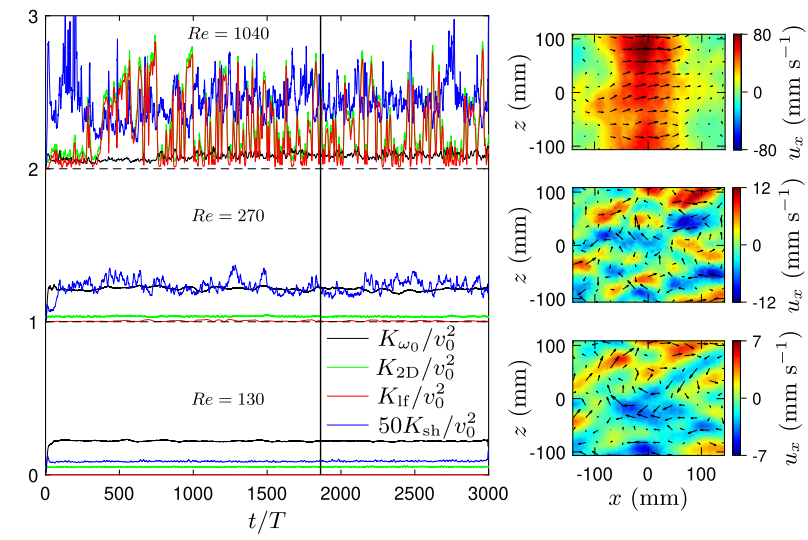

FIG. 3. Time series of the kinetic energies $K_{\omega_{0}}$ of the forced mode, $K_{2 \mathrm{D}}$ of the 2D mode, $K_{\mathrm{lf}}$ of the low-frequency mode, and $K_{\text {sh }}$ of the first subharmonic mode (the latter being multiplied by 50) for $\operatorname{Re}=130 ; 270$ and 1040 and normalized by $v_{0}^{2}=\left(A \omega_{0}\right)^{2}$. The forcing starts at $t=0$. A vertical shift of 1 has been introduced between the curves at different Re. For each Re, the right panel shows the corresponding velocity field at time $t=1830 T$. 
still negligible energy $K_{\text {lf }}$ in the low-frequency geostrophic component. (iii) For $\operatorname{Re}=1040$, the triadic instability first sets in, inducing significant levels of $K_{\mathrm{sh}}$, before the geostrophic mode arises and settles in a strongly fluctuating state with large $K_{2 \mathrm{D}} \simeq K_{\text {lf }}$ [34]. The geostrophic flow thus arises above a threshold value of the driving intensity, as a secondary instability of the triadic instability. While the first triadic instability is in line with the phenomenology of wave turbulence, the system then rapidly finds a shortcut to directly transfer energy to the geostrophic mode.

Quartetic secondary instability.-We propose a generic scenario to explain this shortcut to geostrophy: while resonant triads cannot transfer energy to the slow geostrophic modes, resonant quartets can, as discussed by Smith and Waleffe (SW) [6]. More surprisingly, we will show that these resonant quartets can spontaneously generate geostrophic flows through a "quartetic instability," the four-wave equivalent of the triadic instability. To illustrate this mechanism, we focus on a single resonant triad, and an associated single resonant quartet. They are chosen somewhat arbitrarily, with the goal of providing a simple example of quartetic shortcut to geostrophy.

We thus consider the resonant triad in Fig. 4(a), with wave vectors $\mathbf{k}_{2}=k_{0}[6 ; 0 ;-3], \mathbf{k}_{3}^{\prime}=k_{0}[-18 / 5 ; 0 ;-9 / 5]$, and $\mathbf{k}_{4}^{\prime}=k_{0}[-12 / 5 ; 0 ; 24 / 5]$ and polarities $\left(s_{2}=+1, s_{3}^{\prime}=+1\right.$, $s_{4}^{\prime}=+1$ ) in the conventions of SW (see Supplemental Material [35]). $k_{0}$ is an arbitrary inverse length scale that does not enter the analysis. In a similar fashion to our experiments, we consider a system where wave makers generate waves at frequency $\omega\left(\mathbf{k}_{4}^{\prime}\right)$, energizing wave vector $\mathbf{k}_{4}^{\prime}$ but also many other parallel wave vectors associated with the same frequency [see Fig. 4(a)] [36]. Above a threshold amplitude of the forcing, the system spontaneously transfers some energy from the mode $\mathbf{k}_{4}^{\prime}$ to $\mathbf{k}_{3}^{\prime}$ and $\mathbf{k}_{2}$ through the triadic resonance instability mechanism [21,30]. Consider then the quartet sketched in Fig. 4(b),
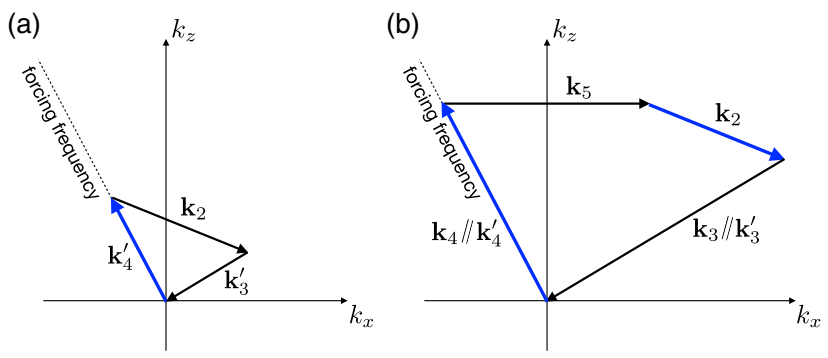

FIG. 4. An illustrative example of the quartetic secondary instability. (a) The triadic instability transfers energy from the mode $\mathbf{k}_{4}^{\prime}$ (at the forcing frequency) to modes at $\mathbf{k}_{2}$ and $\mathbf{k}_{3}^{\prime}$. (b) One can build a resonant quartet by keeping $\mathbf{k}_{2}$, inserting a horizontal wave number $\mathbf{k}_{5}$, and closing the quartet with two wave vectors $\mathbf{k}_{3}$ and $\mathbf{k}_{4}$ parallel to $\mathbf{k}_{3}^{\prime}$ and $\mathbf{k}_{4}^{\prime}$, respectively. $\mathbf{k}_{4}$ is energized by the forcing, while $\mathbf{k}_{2}$ has been energized at step (a). Through a resonant quartet instability, the geostrophic mode $\mathbf{k}_{5}$ then spontaneously emerges, together with $\mathbf{k}_{3}$. built by keeping wave vector $\mathbf{k}_{2}$, inserting a horizontal wave vector $\mathbf{k}_{5}=k_{0}[8 ; 0 ; 0]$, and closing the quartet with two wave vectors $\mathbf{k}_{3}=k_{0}[-10 ; 0 ;-5]$ and $\mathbf{k}_{4}=$ $k_{0}[-4 ; 0 ; 8]$ parallel to $\mathbf{k}_{3}^{\prime}$ and $\mathbf{k}_{4}^{\prime}$, respectively. The quartet $\left(\mathbf{k}_{2}, \mathbf{k}_{3}, \mathbf{k}_{4}, \mathbf{k}_{5}\right)$ happens to be the one considered by Smith and Waleffe [6]. This quartet is resonant for the mode polarities $\left(s_{2}, s_{3}, s_{4}, s_{5}\right)=(+1,+1,+1,-1)$, which we adopt in the following. The mode $\mathbf{k}_{4}$ is at frequency $\omega\left(\mathbf{k}_{4}\right)=\omega\left(\mathbf{k}_{4}^{\prime}\right)$ and is also energized by the forcing, while the mode $\mathbf{k}_{2}$ has been energized by the triadic instability at the previous step. We now show that the mode $\mathbf{k}_{3}$ and the geostrophic mode $\mathbf{k}_{5}$ can emerge spontaneously through a quartetic instability. Denoting as $b_{i}$ the amplitude of the mode of wave vector $\mathbf{k}_{i}$ with the polarity $s_{i}$, a multipletimescale expansion in the low-Rossby-number limit yields the following quartetic evolution equations:

$$
\begin{gathered}
\left(\partial_{t}+\nu k_{3}^{2}\right) b_{3}=C_{\mathbf{k}_{3} ; \mathbf{k}_{2} ; \mathbf{k}_{4} ; \mathbf{k}_{5}}^{+1,+1,+1,-1} \overline{b_{2}} \overline{b_{4}} \overline{b_{5}}, \\
\left(\partial_{t}+\mu+\nu k_{5}^{2}\right) b_{5}=C_{\mathbf{k}_{5} ; \mathbf{k}_{2} ; \mathbf{k}_{3} ; \mathbf{k}_{4}}^{-1,+1,+1, \overline{b_{2}}} \overline{b_{3}} \overline{b_{4}},
\end{gathered}
$$

where an overbar denotes the complex conjugate and the $\mu$-term mimics bottom-drag acting on the geostrophic mode. The details of the asymptotic expansion are deferred to the Supplemental Material [35], as well as the expressions of the ( $\mu$ - and $\nu$-independent) quartetic coefficients

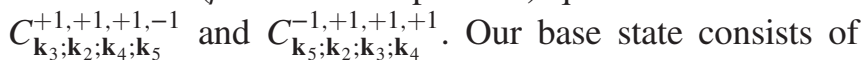
time-independent finite amplitudes $b_{2}$ and $b_{4}$, energized, respectively, by the forcing and by the triadic instability. We consider infinitesimal perturbations of the modes 3 and $5, b_{3}(t) \ll 1$ and $b_{5}(t) \ll 1$. Upon looking for perturbations evolving as $e^{\gamma t}$ and neglecting the dissipative terms, Eqs. (1) and (2) yield

$$
\gamma= \pm \sqrt{C_{\mathbf{k}_{3} ; \mathbf{k}_{2} ; \mathbf{k}_{4} ; \mathbf{k}_{5}}^{+1,+1,+1,-1} \times \bar{C}_{\mathbf{k}_{5} ; \mathbf{k}_{2} ; \mathbf{k}_{3} ; \mathbf{k}_{4}}^{-1,+1,+1,1}}\left|b_{2}\right|\left|b_{4}\right| .
$$

Because $C_{\mathbf{k}_{3} ; \mathbf{k}_{2} ; \mathbf{k}_{4} ; \mathbf{k}_{5}}^{+1,+1,1,-1} \times \bar{C}_{\mathbf{k}_{5} ; \mathbf{k}_{2} ; \mathbf{k}_{3} ; \mathbf{k}_{4}}^{-1,+1,+1,+1}>0$ (see Supplemental Material [35]), waves at $\mathbf{k}_{3}$ and $\mathbf{k}_{5}$ grow exponentially: a geostrophic flow emerges spontaneously through this quartetic instability. When the damping terms are retained, the amplification gain (3) must overcome viscous and frictional damping. This occurs above a threshold value of the product $\left|b_{2}\right|\left|b_{4}\right|$, which we determine by performing a similar linear stability analysis on the full equations (1) and (2), before setting $\gamma$ to zero. This yields the instability criterion

$$
\left|b_{2}\right|\left|b_{4}\right| \geq \sqrt{\frac{\nu k_{3}^{2}\left(\mu+\nu k_{5}^{2}\right)}{C_{\mathbf{k}_{3} ; \mathbf{k}_{2} ; \mathbf{k}_{4} ; \mathbf{k}_{5}}^{+1,+1,-1} \times \bar{C}_{\mathbf{k}_{5} ; \mathbf{k}_{2} ; \mathbf{k}_{3} ; \mathbf{k}_{4}}^{-1,+1,+1,+1}}} .
$$

In other words, the system needs to be sufficiently above the threshold of the triadic instability, with large enough $\left|b_{2}\right|$, to trigger the quartetic secondary instability. 


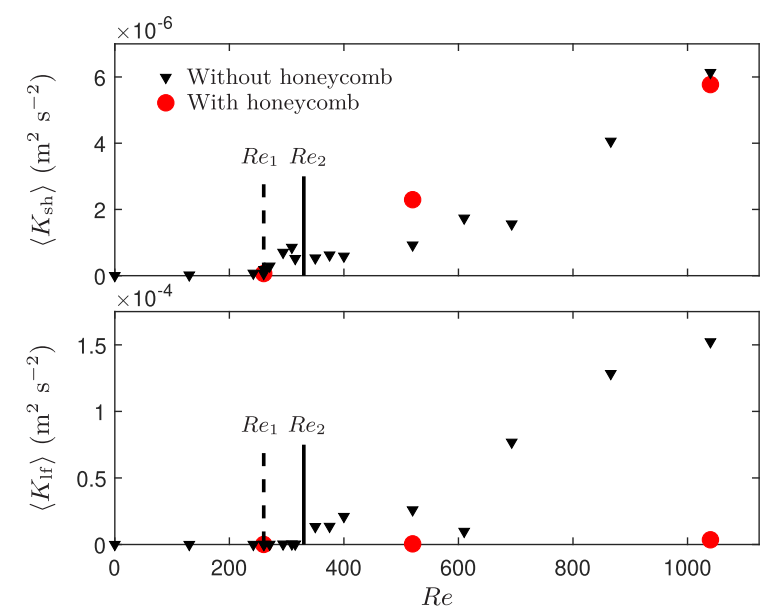

FIG. 5. $\left\langle K_{\mathrm{sh}}\right\rangle$ and $\left\langle K_{\mathrm{lf}}\right\rangle$ as functions of Re. The threshold Reynolds number $\operatorname{Re}_{2}$ for the emergence of geostrophy is $25 \%$ higher than the threshold $\operatorname{Re}_{1}$ of the triadic instability when the bottom boundary is smooth. $\mathrm{Re}_{2}$ is shifted to prohibitively large values when the bottom boundary is made rough using a honeycomb structure. The error bars on $\left\langle K_{\mathrm{sh}}\right\rangle$ are comparable to the size of the symbols. The error bars on $\left\langle K_{\mathrm{lf}}\right\rangle$ are comparable to the size of the symbols for $\operatorname{Re} \leq 600$, and around $\pm 15 \%$ for $\operatorname{Re}>600$.

To confront this scenario to the experimental data, we plot in Fig. 5 the time-averaged kinetic energies $\left\langle K_{\text {sh }}\right\rangle$ and $\left\langle K_{\text {If }}\right\rangle$ as functions of Re. The primary triadic instability arises above a threshold value $\operatorname{Re}_{1} \simeq 260$, where $\left\langle K_{\mathrm{sh}}\right\rangle$ departs from zero, while the threshold of the quartetic instability is $\operatorname{Re}_{2} \simeq 330$, where $\left\langle K_{\text {lf }}\right\rangle$ departs from zero. One may notice that $\left\langle K_{\text {lf }}\right\rangle$ has a nonmonotonic behavior at higher Re. This feature goes beyond the scope of the present model, which describes the immediate vicinity of the bifurcation points only. An interesting extension of the model could be to focus on scale separation between wave motion and large-scale geostrophic flow, to capture the four-wave instability through mean-field coefficients [37-39], in the spirit of Refs. [40,41].

Despite its simplicity - a triad inside a vertical plane, and an associated quartet inside that same plane- the model successfully captures the sequence of bifurcations observed in the experiment: a primary triadic instability followed by a secondary quartetic instability, which triggers the emergence of geostrophy. The frequency of the forced waves in the theoretical model being close to the frequency of the forced waves in the laboratory experiment (respectively, $\omega\left(\mathbf{k}_{4}\right)=\omega\left(\mathbf{k}_{4}^{\prime}\right)=0.89 \times 2 \Omega$ and $\omega_{0}=0.84 \times 2 \Omega$ ), we can compare quantitatively the various terms arising in the model to their experimental counterparts. We estimate both sides of Eq. (4) at the experimental threshold $\mathrm{Re}_{2}$ where geostrophy emerges: the amplitude of the primary waves $\left|b_{4}\right|$ is approximately $\sqrt{\left\langle K_{\omega_{0}}\right\rangle} \simeq 4.5 \mathrm{~mm} \mathrm{~s}^{-1}$. The square-root of $\left\langle K_{\mathrm{sh}}\right\rangle$ gives $\left|b_{2}\right| \simeq 0.7 \mathrm{~mm} \mathrm{~s}^{-1}$, and the left-hand side of Eq. (4) is around $3.2 \times 10^{-6} \mathrm{~m}^{2} \mathrm{~s}^{-2}$. The
Ekman friction term is here comparable to the viscous one, and the wave vectors have comparable lengths. We thus estimate the numerator of the right-hand side of Eq. (4) as simply $\nu k_{4}^{2}$. Substituting $k_{4}=\sqrt{80} k_{0}$ and $C_{\mathbf{k}_{3} ; \mathbf{k}_{2} ; \mathbf{k}_{4} ; \mathbf{k}_{5}}^{+1,+1,+1,-1} \times$ $\bar{C}_{\mathbf{k}_{5} ; \mathbf{k}_{2} ; \mathbf{k}_{3} ; \mathbf{k}_{4}}^{-1,+1,1,+1} \simeq 726 k_{0}^{4} / \Omega^{2}$ (computed in the Supplemental Material [35]), the right-hand side of Eq. (4) is estimated as $80 \nu \Omega / \sqrt{726} \simeq 5.6 \times 10^{-6} \mathrm{~m}^{2} \mathrm{~s}^{-2}$. We conclude that the two sides of Eq. (4) have comparable magnitudes at $\mathrm{Re}_{2}$, which confirms the expected balance between quartetic amplification and damping terms at threshold.

Beyond these order of magnitude estimates, fully quantitative predictions would require an exact knowledge of the base flow, together with an optimization of the bifurcation threshold over all possible combinations of resonant triads and associated resonant quartets, a challenging task in general. Nevertheless, we can test the qualitative predictions of the theory further: we performed three complementary experiments with a honeycomb structure at the bottom of the tank $($ mesh $=1.7 \mathrm{~cm}$, height $=2 \mathrm{~cm})$. We first sent an inertial-wave beam towards this rough bottom boundary and observed that it reflects with negligible losses: the honeycomb structure has little effect on wavelike motion. By contrast, we expect it to induce a strong damping of the geostrophic modes, through a drastic increase in bottom drag. In some sense, this honeycomb structure is an experimental means of achieving the specific damping of the geostrophic modes included numerically by Le Reun et al. [32]. In line with these expectations, we observe in Fig. 5 that the onset of the triadic instability remains unaffected, while the threshold of the secondary quartetic instability is shifted to prohibitively large values of Re [42]. These observations are qualitatively captured by expression (4) at large $\mu$, this large $\mu$ being a rough modelization of the possibly turbulent and quadratic bottom drag induced by the honeycomb structure. This provides further experimental evidence that the subharmonic waves and the geostrophic flow appear through distinct instabilities.

Discussion.-Although our study focuses on rotating turbulence, the phenomenology probably holds for the various anisotropic fluid systems that can be decomposed into fast wave modes and a slow manifold: Rossby waves and zonal flows on the beta plane, internal gravity waves and shear flows in stratified fluids, etc. As a matter of fact, the ability of quartets of inertial waves to transfer energy to the geostrophic modes was soon identified by SW, based on previous work by Newell [43] showing that quartets of Rossby waves can force zonal flows. These studies focus on the direct forcing of the slow manifold by three preexisting waves. By contrast, here we force waves at a single frequency. Even in that case, however, our experimental results show that the system efficiently transfers energy to the $2 \mathrm{D}$ flow through a quartetic instability. A somewhat similar situation was recently reported by Le Reun et al. [44]. In their experiment, the libration of an ellipsoid induces a $2 \mathrm{D}$ base flow that is unstable to 
inertial-wave motion. As one increases the driving amplitude, the system first transfers energy to secondary inertialwave modes through triadic resonances before inducing a geostrophic vortex on top of the base librating flow. The authors conclude that this geostrophic vortex probably emerges because the Rossby number is only moderately low, although they also invoke the possible role of an instability described by Kerswell in cylindrical geometry [45]. Although it was not identified as such at the time, we believe that Kerswell's instability may very well be underpinned by the present four-wave mechanism: the corresponding quartet would consist of two wave modes and two geostrophic ones, suggesting a scenario where geostrophy arises through a direct quartetic instability (as opposed to a secondary one). While it is difficult to discriminate between the direct and secondary quartetic instability scenarios experimentally, we expect the direct instability to be more strongly stabilized by bottom friction than the secondary instability, because it involves two geostrophic modes. By contrast, the secondary instability involves a single geostrophic mode, hence a single factor $\sqrt{\mu}$ on the right-hand side of Eq. (4).

The quartetic instability induces a transfer of energy to the slow modes, thus restricting the domain of validity of WTT. Indeed, the relation (4) indicates that this instability sets in when the Ekman number is reduced at fixed Rossby number. The only hope to observe weak wave turbulence is then to focus on distinguished limits where the Rossby number goes to zero faster than some power of the Ekman number [32], the precise boundary in parameter space depending on the dominant damping mechanism (bulk viscosity, Ekman friction, etc.). For wave turbulence to develop, this damping mechanism must be dominant at the quartetic order, but negligible at the triadic one. The corresponding parameter range may be too narrow for an experimental study. A more promising approach could be the use of rough boundaries, such as the honeycomb structure we placed at the bottom of the tank, to induce turbulent Ekman layers and preferential damping of the geostrophic flow.

We acknowledge J. Amarni, A. Aubertin, L. Auffray, and R. Pidoux for experimental help. B. G. thanks T. Le Reun, B. Favier, and M. Le Bars for insightful discussions. This work has been supported by the Agence Nationale de la Recherche through Grant "DisET" No. ANR-17-CE300003. B. G. acknowledges support by the European Research Council under Grant Agreement No. 757239. This work was supported by a grant from the Simons Foundation (651461, PPC).

*ppcortet@fast.u-psud.fr

[1] P. Sagaut and C. Cambon, Homogeneous Turbulence Dynamics (Cambridge University Press, Cambridge, England, 2008).
[2] P. A. Davidson, Turbulence in Rotating, Stratified and Electrically Conducting Fluids (Cambridge University Press, Cambridge, England, 2013).

[3] J. Pedlosky, Geophysical Fluid Dynamics (Springer-Verlag, New York, 1987).

[4] H. Greenspan, The Theory of Rotating Fluids (Cambridge University Press, Cambridge, England, 1968).

[5] S. Nazarenko, Wave Turbulence (Springer, Berlin, 2011).

[6] L. M. Smith and F. Waleffe, Phys. Fluids 11, 1608 (1999).

[7] E. J. Hopfinger, F. K. Browand, and Y. Gagne, J. Fluid Mech. 125, 505 (1982).

[8] L. Jacquin, O. Leuchter, C. Cambon, and J. Mathieu, J. Fluid Mech. 220, 1 (1990).

[9] P. J. Staplehurst, P. A. Davidson, and S. B. Dalziel, J. Fluid Mech. 598, 81 (2008).

[10] C. Lamriben, P.-P. Cortet, and F. Moisy, Phys. Rev. Lett. 107, 024503 (2011).

[11] C. N. Baroud, B. B. Plapp, H. L. Swinney, and Z. S. She, Phys. Fluids 15, 2091 (2003).

[12] E. Yarom, Y. Vardi, and E. Sharon, Phys. Fluids 25, 085105 (2013).

[13] E. Yarom and E. Sharon, Nat. Phys. 10, 510 (2014).

[14] E. Yarom, A. Salhov, and E. Sharon, Phys. Rev. Fluids 2, 122601 (2017).

[15] A. Campagne, B. Gallet, F. Moisy, and P.-P. Cortet, Phys. Fluids 26, 125112 (2014).

[16] A. Campagne, B. Gallet, F. Moisy, and P.-P. Cortet, Phys. Rev. E 91, 043016 (2015).

[17] A. Campagne, N. Machicoane, B. Gallet, P.-P. Cortet, and F. Moisy, J. Fluid Mech. 794, R5 (2016).

[18] B. Gallet, J. Fluid Mech. 783, 412 (2015).

[19] A. M. M. Manders and L. R. M. Maas, J. Fluid Mech. 493, 59 (2003).

[20] L. Messio, C. Morize, M. Rabaud, and F. Moisy, Exp. Fluids 44, 519 (2008).

[21] G. Bordes, F. Moisy, T. Dauxois, and P.-P. Cortet, Phys. Fluids 24, 014105 (2012).

[22] M. Duran-Matute, J.-B. Flór, F. S. Godeferd, and C. JauseLabert, Phys. Rev. E 87, 041001(R) (2013).

[23] N. Machicoane, P.-P. Cortet, B. Voisin, and F. Moisy, Phys. Fluids 27, 066602 (2015).

[24] M. Brunet, T. Dauxois, and P.-P. Cortet, Phys. Rev. Fluids 4, 034801 (2019).

[25] P.-P. Cortet, C. Lamriben, and F. Moisy, Phys. Fluids 22, 086603 (2010).

[26] J. Boisson, D. Cébron, F. Moisy, and P.-P. Cortet, Europhys. Lett. 98, 59002 (2012).

[27] S. A. Triana, D. S. Zimmerman, and D. P. Lathrop, J. Geophys. Res. 117, B04103 (2012).

[28] S. Joubaud, J. Munroe, P. Odier, and T. Dauxois, Phys. Fluids 24, 041703 (2012).

[29] H. Scolan, E. Ermanyuk, and T. Dauxois, Phys. Rev. Lett. 110, 234501 (2013).

[30] C. R. Koudella and C. Staquet, J. Fluid Mech. 548, 165 (2006).

[31] L. Jouve and G. I. Ogilvie, J. Fluid Mech. 745, 223 (2014).

[32] T. Le Reun, B. Favier, A. J. Barker, and M. Le Bars, Phys. Rev. Lett. 119, 034502 (2017). 
[33] For larger Re, they move farther away from $\omega_{0}^{*} / 2$. They also widen in frequency while their overall energy content increases with Re, see Fig. 5.

[34] These fluctuations are due to the geostrophic vortices coming in and out of the PIV plane.

[35] See Supplemental Material at http://link.aps.org/supplemental/ 10.1103/PhysRevLett.124.124501 for the derivation of the quartetic interaction coefficients.

[36] In agreement with the dispersion relation of inertial waves, $\omega=s 2 \mathbf{k} \cdot \mathbf{\Omega} /|\mathbf{k}|$.

[37] U. Frisch, Z. S. She, and P. L. Sulem, Physica 28D, 382 (1987).
[38] B. Dubrulle and U. Frisch, Phys. Rev. A 43, 5355 (1991).

[39] G. I. Sivashinsky, Phys. Fluids A 4, 1608 (1992).

[40] N. Kleeorin and I. Rogachevskii, J. Plasma Phys. 84, 735840303 (2018).

[41] I. Rogachevskii and N. Kleeorin, Phys. Rev. E 100, 063101 (2019).

[42] See the associated spectrum in Fig. 2, where the zerofrequency geostrophic peak has disappeared.

[43] A. C. Newell, J. Fluid Mech. 35, 255 (1969).

[44] T. Le Reun, B. Favier, and M. Le Bars, J. Fluid Mech. 879, 296 (2019).

[45] R. R. Kerswell, J. Fluid Mech. 382, 283 (1999). 